\title{
Species of Bursaphelenchus Fuchs, 1937 (Nematoda: Parasitaphelenchidae) associated with maritime pine in Portugal
}

\author{
Ana Catarina PEnAS ${ }^{1}$, Petra Correia ${ }^{2}$, Maria Antónia Bravo ${ }^{1}$, \\ Manuel MOTA $^{3, *}$ and Rogério TENREIRO ${ }^{2}$ \\ ${ }^{1}$ Departamento de Protecção das Plantas, Estação Agronómica Nacional (EAN), INIA, \\ Quinta do Marquês, 2784-505 Oeiras, Portugal \\ ${ }^{2}$ Departamento de Biologia Vegetal, Centro de Genética e Biologia Molecular, \\ Faculdade de Ciências da Universidade de Lisboa, Campo Grande, 1749-016 Lisboa, Portugal \\ ${ }^{3}$ NemaLab/ICAM, Departamento de Biologia, Universidade de Évora, 7002-554 Évora, Portugal
}

Received: 19 January 2004; revised: 22 April 2004 Accepted for publication: 29 April 2004

\begin{abstract}
Summary - Species of Bursaphelenchus associated with maritime pine, Pinus pinaster, from Portugal - within and outside the quarantine restricted demarcated zone of B. xylophilus - are described and characterised both morphologically (LM and SEM) and with the use of molecular biology (ITS-RFLP). A new staining method for spicules is proposed. Species include B. hellenicus, B. hylobianum, B. leoni, B. pinophilus, B. sexdentati, B. tusciae, B. teratospicularis, B. xylophilus and Bursaphelenchus sp. 1. Bursaphelenchus hylobianum was collected from the insect Hylobius sp. The most frequent species in the demarcated zone, besides B. xylophilus, was Bursaphelenchus sp. 1. Morphological characterisation is compared with the original descriptions and discussed. The differentiation between $B$. pinophilus and B. sexdentati is not clear in the literature and is discussed. Since differentiation of B. xylophilus (mucronate form) from B. mucronatus, and B. pinophilus from B. sexdentati, as well as their juvenile forms, is almost im possible on the basis of morphological features, a molecular approach based on ITS-RFLPs was used. Ribosomal DNA containing the 5.8S gene, the internal transcribed spacer region 1 and 2, and partial regions of 18S and 28S gene were amplified by PCR. Restriction profiles of the amplified products generated species-specific differences, leading to the unambiguous identification of isolates belonging to B. xylophilus, B. mucronatus, B. sexdentati, B. tusciae and B. hylobianum.
\end{abstract}

Keywords - diagnostics, identification, ITS-RFLP, morphology, Pinus pinaster, survey.

During a survey conducted within a national Praxis XXI research project no. 11 189/98, 'Survey and study of Bursaphelenchus and other nematode species associated with cerambycid insects in pine trees in Portugal', the pinewood nematode, Bursaphelenchus xylophilus (Steiner \& Buhrer, 1934) Nickle 1970, was detected in March of 1999, associated with maritime pine (Pinus pinaster Aiton). This represented the first report of this important pest and pathogen in Portugal and Europe (Mota et al., 1999).

Official authorities implemented an intensive survey of the region where the nematode was detected and later expanded it to the rest of the country within a National Programme of Pinewood Nematode Control (PROLUNP, Programa Nacional de Luta contra o Nemátode do Pinheiro - http://www.dgf.min-agricultura.pt/prolunp/html/ home-final.htm). As a result, this A1 quarantine organism was confirmed as being restricted within an area in the Setúbal region to the southeast of Lisbon. This infested area has been precisely delimited by annual surveys and a buffer zone, about $20 \mathrm{~km}$ wide, was established and included in a demarcated zone subject to restrictive quarantine measures. Since then, in addition to an intensive annual survey of the demarcated zone, the survey programme has also been carried out in the rest of the country (Mota \& Vieira, 2003).

About 25 species of Bursaphelenchus associated with conifers have been reported in Europe (Braasch, 2001; Mota \& Vieira, 2004). Some of these species, such as B. mucronatus Mamiya \& Enda, 1979 and B. xylophilus, display similar morphological features which may con-

\footnotetext{
*Corresponding author, e-mail: mmota@uevora.pt
} 
found precise identification, a crucial step in establishing quarantine measures.

Morphology is an essential component of nematode differentiation and, in many cases, provides a rapid and unambiguous species diagnosis. However, this identification is highly dependent on the experience and interpretative skills of the researcher. In some groups of nematodes, morphological characters are only a first approach and require confirmation by more accurate methods. Differences in nucleic acid sequences, as revealed by means of molecular biology techniques such as ITS-RFLP (Hoyer et al., 1998; Braasch et al., 1999), may help to characterise each species and complement its morphological description. Furthermore, DNA-based methods provide an attractive solution to problems associated with morphological identification, since they do not rely on the expression of the genome and are independent of environmental influence or developmental stage.

The purpose of this research was to survey and identify the Bursaphelenchus species associated with maritime pine in Portugal by means of morphological and molecular analysis (PCR-ITS RFLP).

\section{Materials and methods}

During the annual surveys carried out by PROLUNP, a total of 4810 samples of pine wood material from trees displaying symptoms of pine wilt disease, corresponding to a small proportion of all samples, were examined for Bursaphelenchus species in the nematology laboratory of EAN. Nematodes were extracted using a modified Baermann funnel technique. In addition, 62 specimens of Hylobius sp., collected from Pinus pinaster, were crushed in a Syracuse dish and examined under a stereoscopic microscope for possible Bursaphelenchus dauer juveniles. These were inoculated into $15 \mathrm{~cm}$ pine branch segments and Botrytis cinerea Petri dishes, and incubated for 1 month at $26^{\circ} \mathrm{C}$.

Nematode identification was based on observations of the main morphological characters, particularly vulval flap, shape of spicules, female tail (Braasch, 2001) and head shape using light (LM) and scanning electron microscopy (SEM). For spicule observation, a new staining method was tested with the use of Rotring ${ }^{\circledR}$ Brilliant Ultramarine Blue ink (stock solution $=$ one $0.5 \mathrm{ml}$ cartridge in $20 \mathrm{ml}$ of acetic acid; prepare a final solution by diluting $1 \mathrm{ml}$ of stock solution in $10 \mathrm{ml}$ of lactophenol). Live nematodes were transferred to a drop of this solution and heated briefly over an alcohol lamp. This staining method was applied to B. hellenicus Skarmoutsos, Braasch \& Michalopoulou, 1998 and B. sexdentati which have inconspicuous spicules. For SEM studies, nematodes were fixed in a mixture of $4 \%$ gluteraldehyde $/ 2 \%$ formaldehyde for several days, post-fixed in $2 \% \mathrm{OsO}_{4}$ overnight, dehydrated in an ethanol series, critical point dried and sputter coated with gold (Eisenback, 1985). Observations were made with a Jeol 35 SEM.

After extraction from wood, aliquots of one to ten nematodes were stored for DNA extraction. Nematodes were heated at $95^{\circ} \mathrm{C}$ for $5 \mathrm{~min}$, homogenised on a glass slide with a micro pestle (Eppendorf $\left.{ }^{\circledR}\right)$ and DNA obtained using the DNeasy Tissue Kit (Quiagen $\left.{ }^{\circledR}\right)$. This procedure was applied to different nematode life stages, namely adult, dauer juvenile, propagative juvenile and resistant juvenile.

The ITS regions of rDNA were amplified using primers F194 and P5368 as described by Ferris et al. (1993) and Vrain (1993), respectively. All polymerase chain reactions were performed in a final volume of $50 \mu \mathrm{l}$ using $10 \mathrm{ng} / \mu \mathrm{l}$ of template DNA, $1 \mu \mathrm{M}$ of each primer, $0.2 \mu \mathrm{M}$ of dNTPs (Invitrogene ${ }^{\circledR}$ ), 2 U of Taq DNA polymerase (Invitrogene ${ }^{\circledR}$ ), $1 \times$ Reaction Buffer (Invitrogene ${ }^{\circledR}$ ) and $1.25 \mathrm{mM}$ of $\mathrm{MgCl} 2$ (Invitrogene ${ }^{\circledR}$ ). The reaction mixture was overlaid with sterile mineral oil to prevent evaporation during PCR cycling. A Stratagene ${ }^{\circledR}$ Robocycler was used for amplification and the reaction consisted of one denaturation step at $94^{\circ} \mathrm{C}$ for $1 \mathrm{~min}, 35$ cycles with denaturation at $94^{\circ} \mathrm{C}$ for $1 \mathrm{~min}$, annealing at $51^{\circ} \mathrm{C}$ for $1 \mathrm{~min}$, polymerisation at $72^{\circ} \mathrm{C}$ for $2 \mathrm{~min}$ and a final extension step at $72^{\circ} \mathrm{C}$ for $5 \mathrm{~min}$. After PCR, $5 \mu \mathrm{l}$ of amplified product was analysed by electrophoresis in a $1 \%$ agarose gel. Data analysis was performed using the Kodak ${ }^{\circledR}$ 1D 2.0 system and 100 bp DNA Ladder (Invitrogene ${ }^{\circledR}$ ) as a molecular size marker.

Restriction analysis of ITS regions was performed with AluI, HaeIII and RsaI restriction endonucleases (Invitrogene ${ }^{\circledR}$ ), using an aliquot of $4 \mu 1$ of the PCR product and $10 \mathrm{U}$ of each enzyme, according to the manufacturer's instructions. Fragments were resolved by electrophoresis in a $2 \%$ agarose gel and data were analysed as described above.

\section{Results}

The geographic distribution of Bursaphelenchus species in Portugal is presented in Table 1 and Figure 1. Morphological identification was based on original and other descriptions. The species are illustrated by light and scan- 
Table 1. Origin and location of Bursaphelenchus species in Portugal.

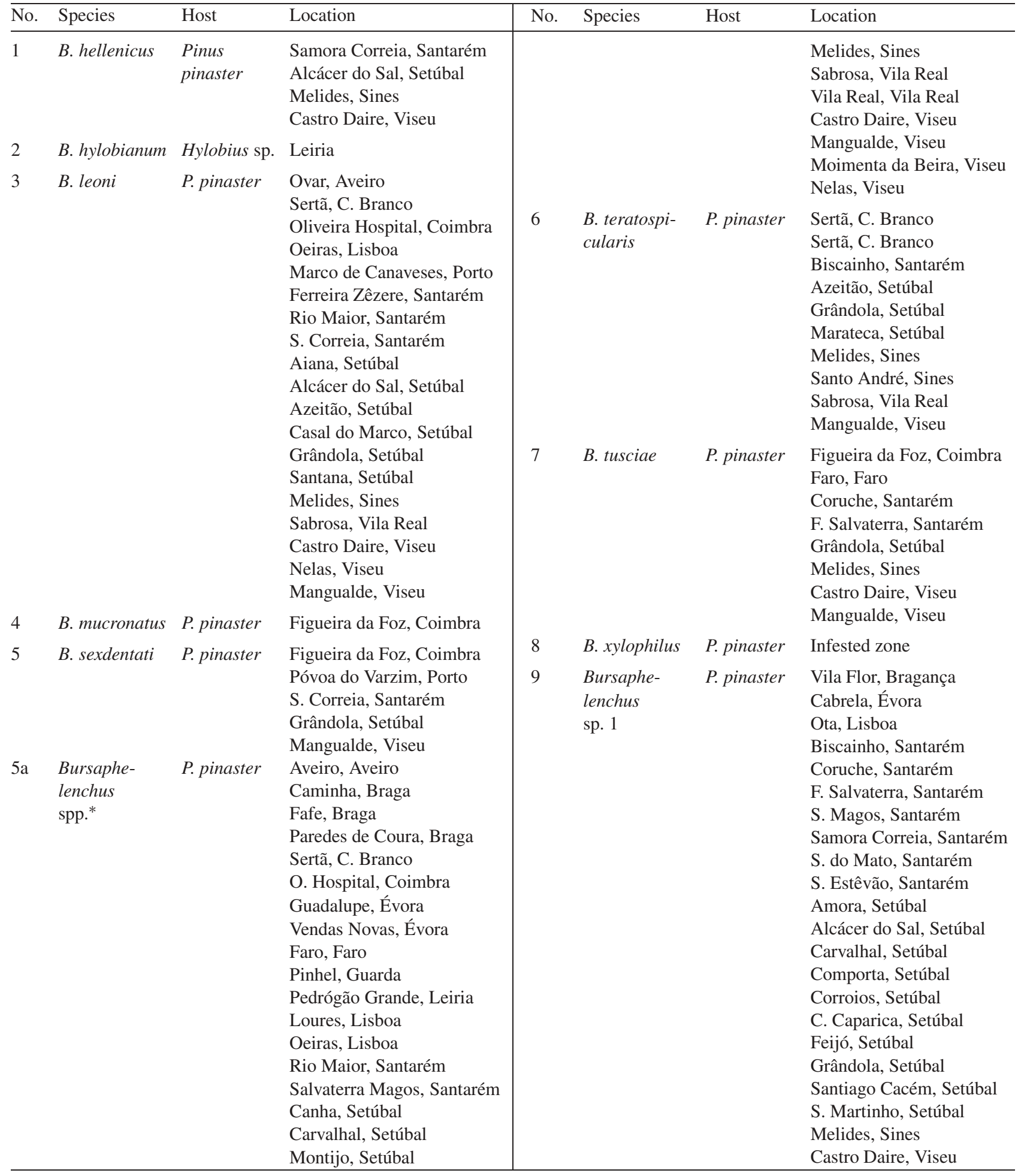

\footnotetext{
${ }^{*}$ B. pinophilus or B. sexdentati (subject to confirmation; see Discussion for more details).
} 


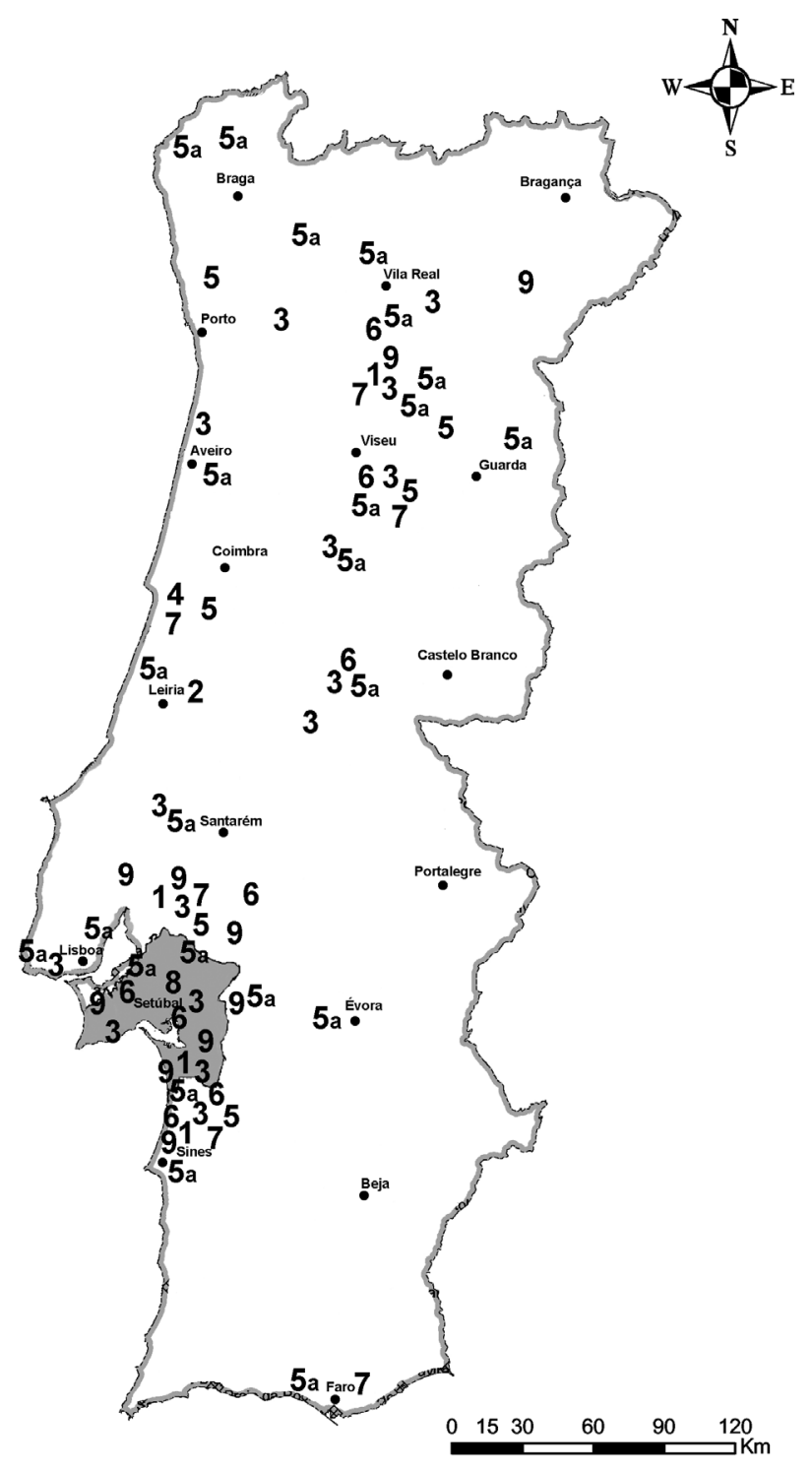

Fig. 1. Occurrence of Bursaphelenchus species in continental Portugal. 1: B. hellenicus; 2: B. hylobianum; 3: B. leoni; 4: B. mucronatus; 5: B. sexdentati; 5a: Bursaphelenchus spp.; 6: B. teratospicularis; 7: B. tusciae; 8: B. xylophilus; 9: Bursaphelenchus $s p .1$ (see Table 1 for details of locations).

ning electron microscopy (Figs 2-10). Besides B. xylophilus (Fig. 9), other Bursaphelenchus species were collected and identified from pine wood, namely: $B$. hellenicus (Fig. 2); B. leoni Baujard, 1980 (Fig. 4); B. mucronatus (Fig. 5); B. teratospicularis Kakuliya \& Devdariani, 1965 (Fig. 7); B. tusciae Ambrogioni \& Palmisano, 1998 (Fig. 8); B. sexdentati Rühm, 1960 (Fig. 6) and Bursaphelenchus sp. 1 (Fig. 10). The presence of B. pinophilus
Brzeski \& Baujard, 1997 in Portugal was previously confirmed by Braasch (2001). ITS-RFLP analysis of some populations suspected to be $B$. sexdentati and/or $B$. pinophilus produced the characteristic pattern for $B$. sexdentati. However, it was not always possible to distinguish clearly between $B$. pinophilus and B. sexdentati, the nematodes in such cases being referred to as Bursaphelenchus spp. One of the species found, Bursaphelenchus sp. 1, was very similar to B. pinasteri Baujard, 1980, although it exhibits some characters, such as head and spicule shape and number of incisures in lateral fields, identical to B. hofmanni Braasch, 1998. Morphological characters used to diagnose the different Bursaphelenchus species are listed and compared in Table 2.

The staining method with blue ink allowed the spicule shape of B. hellenicus and B. sexdentati to be clearly seen (Figs 2, 6).

The number of samples in which each Bursaphelenchus species was found is presented in Table 3. Populations levels were usually low. Apart from B. xylophilus, the most frequent species in the Demarcated Zone was Bursaphelenchus sp. 1, while B. mucronatus was only found in one sample.

Nine Hylobius sp. contained dauer juveniles of Bursaphelenchus sp. under the elytra. Adult nematodes collected from $B$. cinerea plates and pine branches, previously inoculated with these dauer juveniles, were identified as B. hylobianum Korenchenko, 1980 (Fig. 3). As in the original description from Russia (Korenchenko, 1980), this nematode was found in Portugal associated with Hylobius beetles.

In addition to morphological observations, ITS-RFLP analysis was employed for confirmation and differentiation of B. xylophilus, B. mucronatus, B. sexdentati, B. tusciae and $B$. hylobianum. Amplification of ITS regions yielded a single DNA fragment of 950 bp for $B$. $x y$ lophilus, B. mucronatus and B. tusciae, of 1100 bp for $B$. sexdentati and of $1150 \mathrm{bp}$ for $B$. hylobianum. Subsequent analysis of ITS regions with AluI, HaeIII and $R s a \mathrm{I}$ endonucleases produced characteristic restriction profiles that clearly differentiated these five Bursaphelenchus species (Fig. 11). All collected mucronate forms of B. xylophilus individuals displayed the characteristic restriction pattern of this species, thereby allowing for their differentiation from $B$. mисronatus. Similarly, all propagative and resistant juveniles displayed the specific $B$. $x y$ lophilus pattern. Specific patterns were also obtained for dauer juveniles of both B. xylophilus and B hylobianum. 

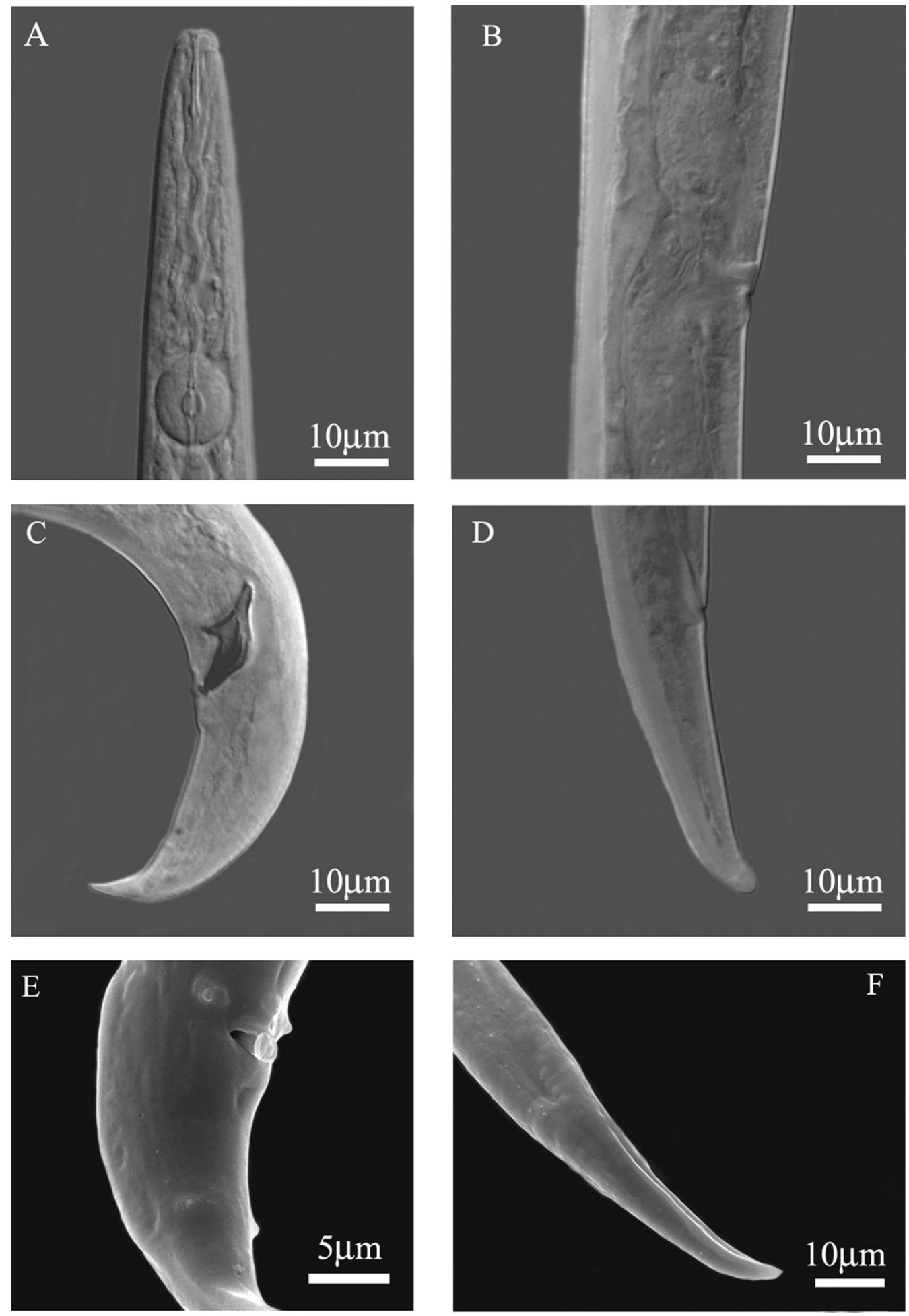

Fig. 2. Bursaphelenchus hellenicus. A: Light micrograph (LM) of anterior region; B: LM of vulval region; C: LM of male tail (stained spicule); D: LM of female tail; E: Scanning electron micrograph (SEM) of male tail showing caudal papillae and protracted cucullus of spicules; F: SEM of female tail. 


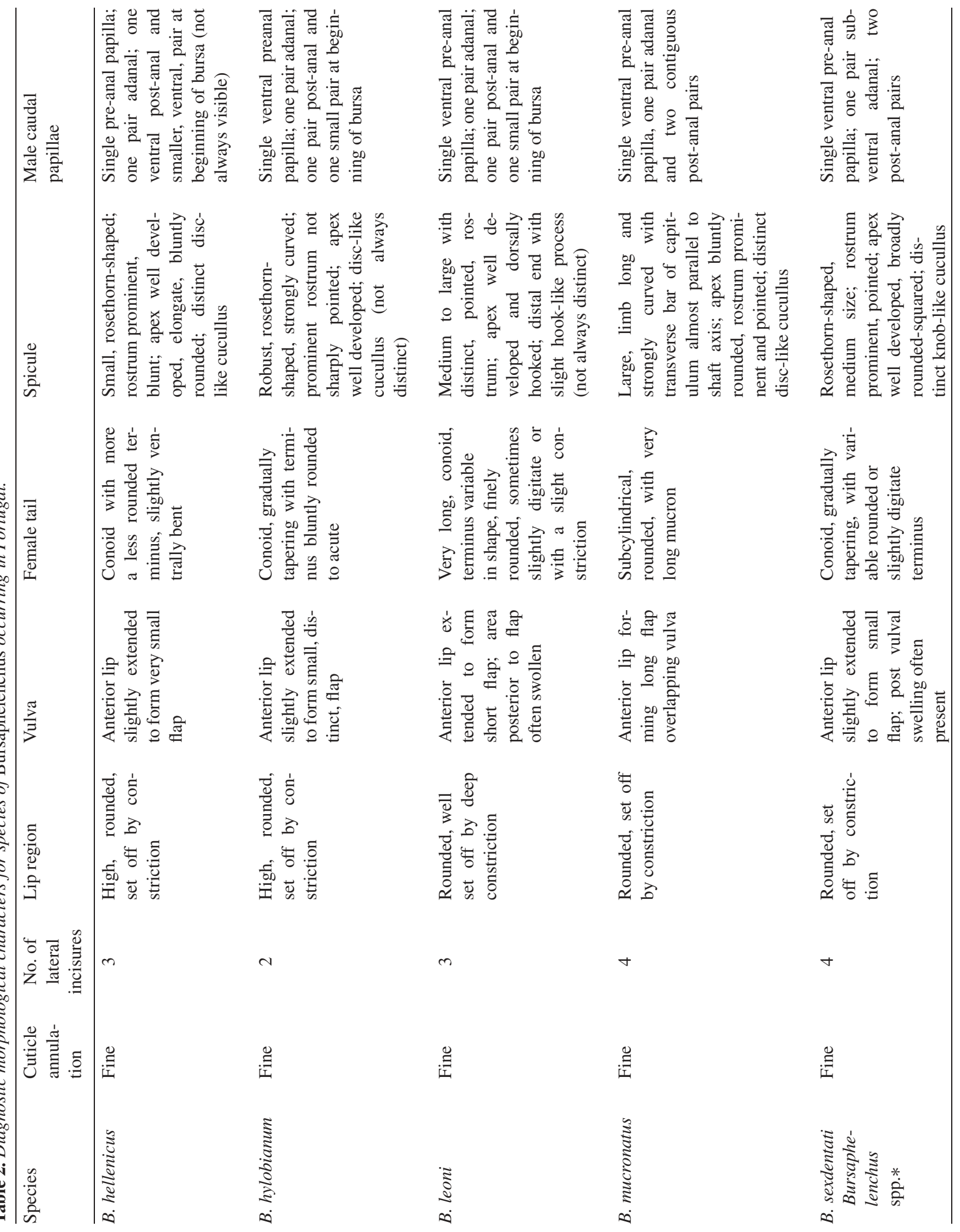




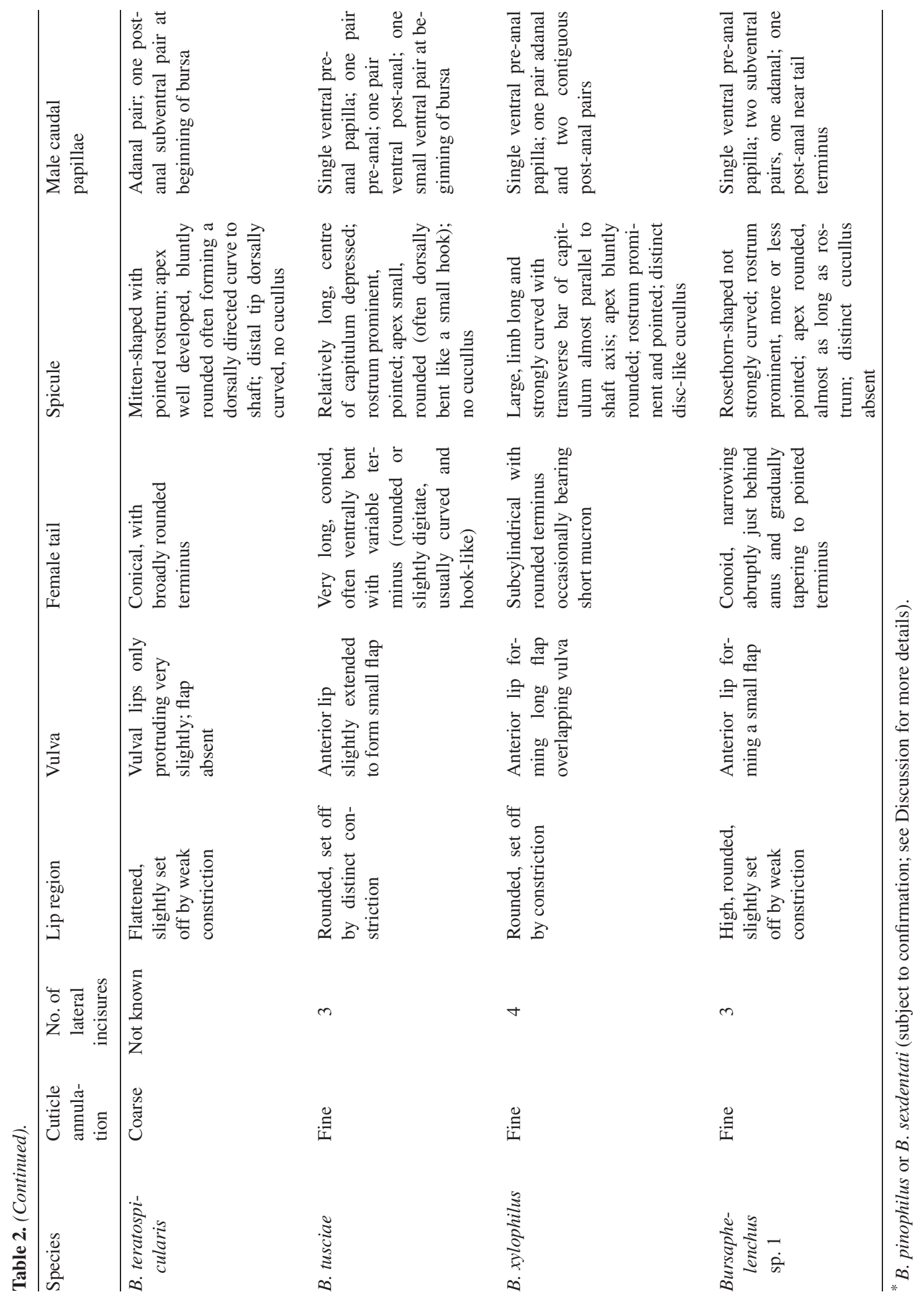



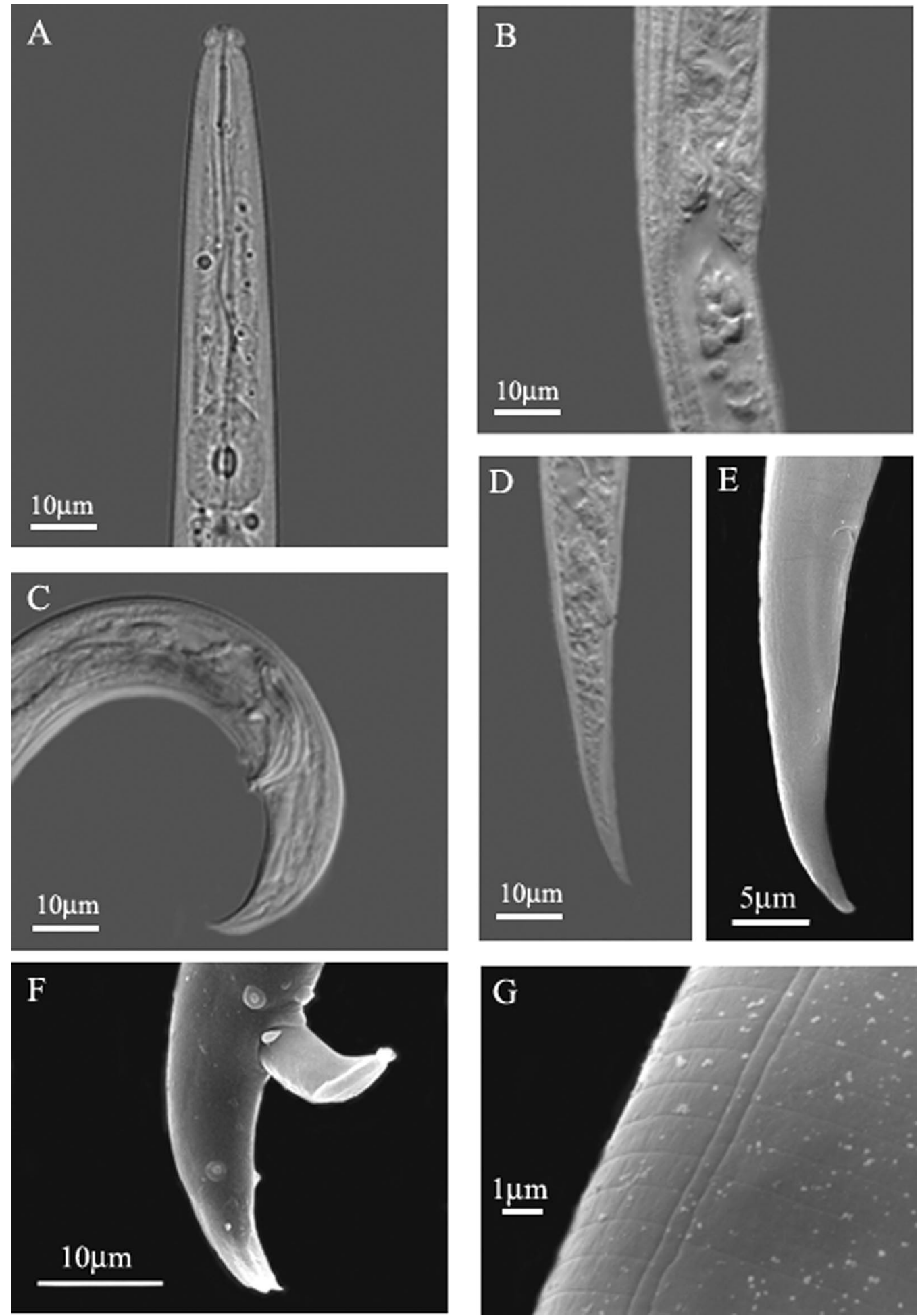

Fig. 3. Bursaphelenchus hylobianum. A: Light micrograph (LM) of anterior region; B: LM of vulval region; $C$ : LM of male tail; D: LM of female tail; E: Scanning electron micrograph (SEM) of female tail; F: SEM of male tail with protracted spicule and caudal papillae; G: SEM of lateral field. 

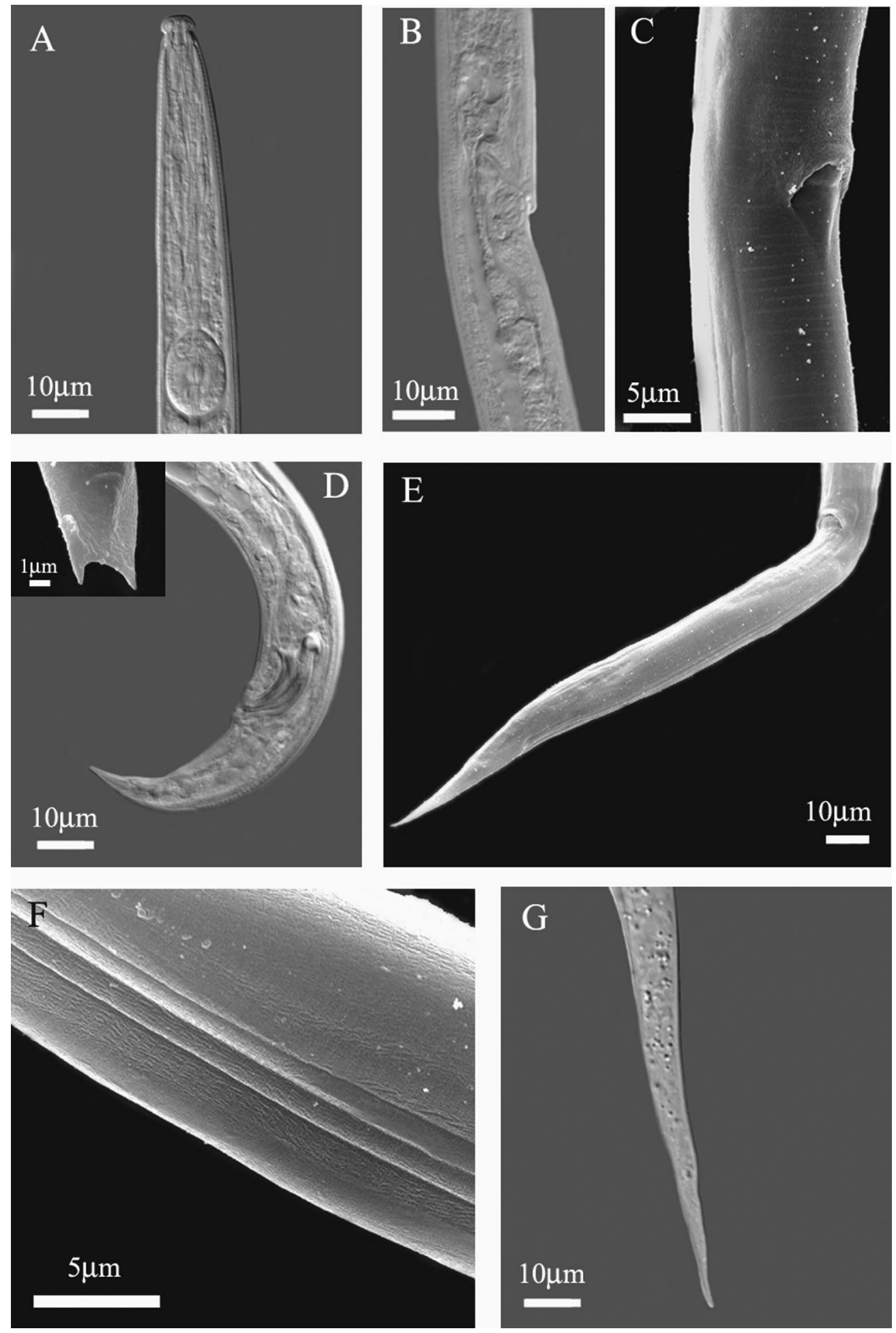

Fig. 4. Bursaphelenchus leoni. A: Light micrograph (LM) of anterior region; B: LM of vulval region; C: Scanning electron micrograph (SEM) of vulval region; D: LM of male tail; inset: SEM of bursa; E: SEM of female tail; F: SEM of lateral field; G: LM of female tail. 

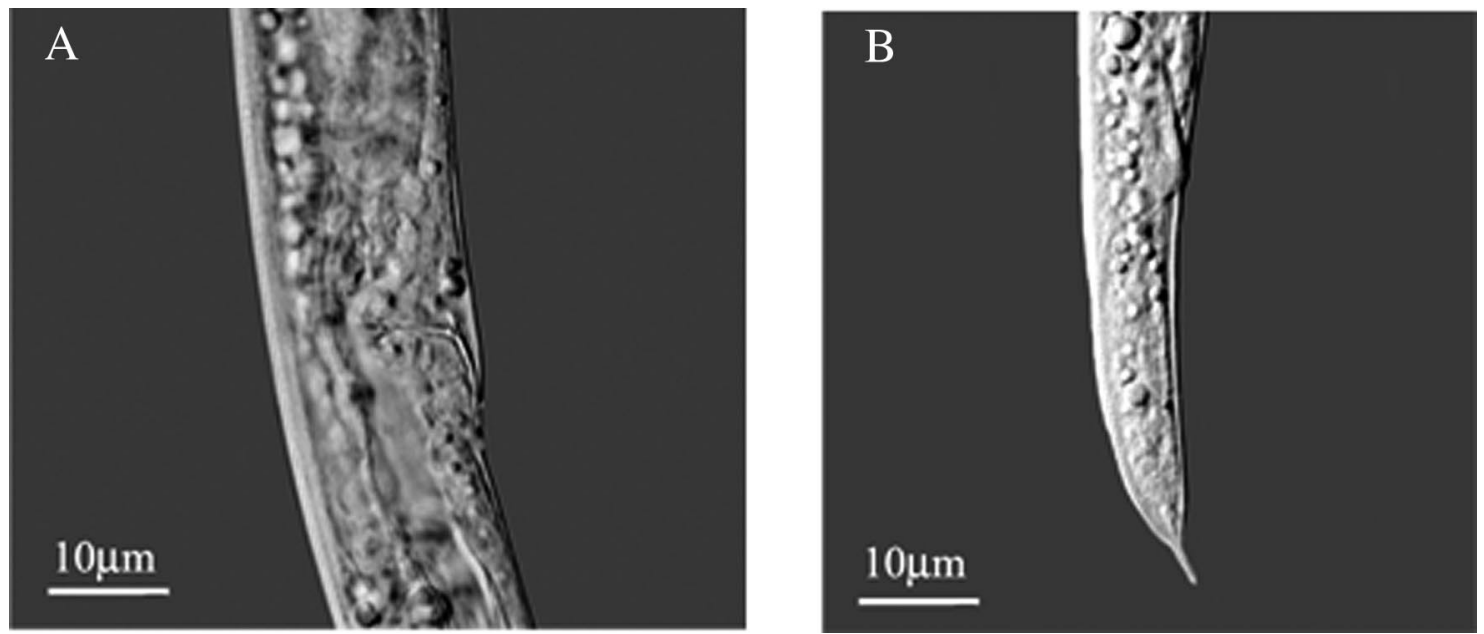

Fig. 5. Bursaphelenchus mucronatus. A: Light micrograph (LM) of vulval region; B: LM of female tail.

Table 3. Number of wood samples containing species of Bursaphelenchus within the National Survey programme and the Demarcated Zone.

\begin{tabular}{lcc}
\hline $\begin{array}{l}\text { Bursaphelenchus } \\
\text { species }\end{array}$ & $\begin{array}{c}\text { National Survey } \\
\text { (1999-2002) }\end{array}$ & $\begin{array}{c}\text { Demarcated Zone } \\
\text { (1999-2003) }\end{array}$ \\
\hline B. hellenicus & 1 & 4681 samples \\
B. hylobianum & 0 & 0 \\
B. leoni & 30 & 15 \\
B. mucronatus & 1 & 0 \\
B. sexdentati & 5 & - \\
Bursaphelenchus & 24 & 17 \\
$\quad$ spp.* & & 11 \\
B. teratospicu- & 4 & 10 \\
$\quad$ laris & 7 & $1422^{* *}$ \\
B. tusciae & 0 & 134 \\
B. xylophilus & 3 & \\
Bursaphelenchus & & \\
$\quad$ sp. 1 & &
\end{tabular}

* $B$. pinophilus or $B$. sexdentati (subject to confirmation; see Discussion for more details).

** Results of a partial survey; for more detailed information see: http://www.dgf.min-agricultura.pt/prolunp/html/home-final.htm

\section{Discussion}

The greater number of Bursaphelenchus species found in northern and central Portugal reflect the higher density of maritime pine forest from those regions whereas the large number of species within the demarcated zone simply reflects a more intensive survey.
Tail and head shape, appearance of the vulval region and particularly the shape and comparative size of the spicules were sufficient for the separation of most species. The presence of round-tailed females with a well developed vulval flap, together with males having the typical spicule shape, provided a definitive morphological identification for B. xylophilus.

Differentiation based solely on morphological characters is not reliable for some species. Some Portuguese B. xylophilus populations have mucronate-tailed females, a feature which may result in confusion of this species with $B$. mucronatus. ITS-RFLP analysis, however, discriminates the mucronate form of B. xylophilus from B. mucronatus (Tarès et al., 1992).

Concerning the differentiation of the species B. sexdentati and B. pinophilus, Rühm (1960), in the original description of $B$. sexdentati, did not mention the presence of a cucullus. Brzeski and Baujard (1997) did not diagnose $B$. pinophilus in comparison to $B$. sexdentati, although Braasch (2001) distinguished the two species on the basis of the presence of a cucullus in B. pinophilus. However, using SEM techniques, Ambrogioni and Caroppo (1998) showed B. sexdentati to have a distinct cucullus. Although, most of our populations exhibited spicules with a distinct cucullus, something which would seem to indicate that they belong to $B$. pinophilus, ITS-RFLP analysis of these same populations produced the characteristic pattern for B. sexdentati. In addition to clarifying morphological identification of nematodes, this molecular method also facilitates the identification of juvenile stages of Bursaphelenchus species, thereby eliminating the need for 

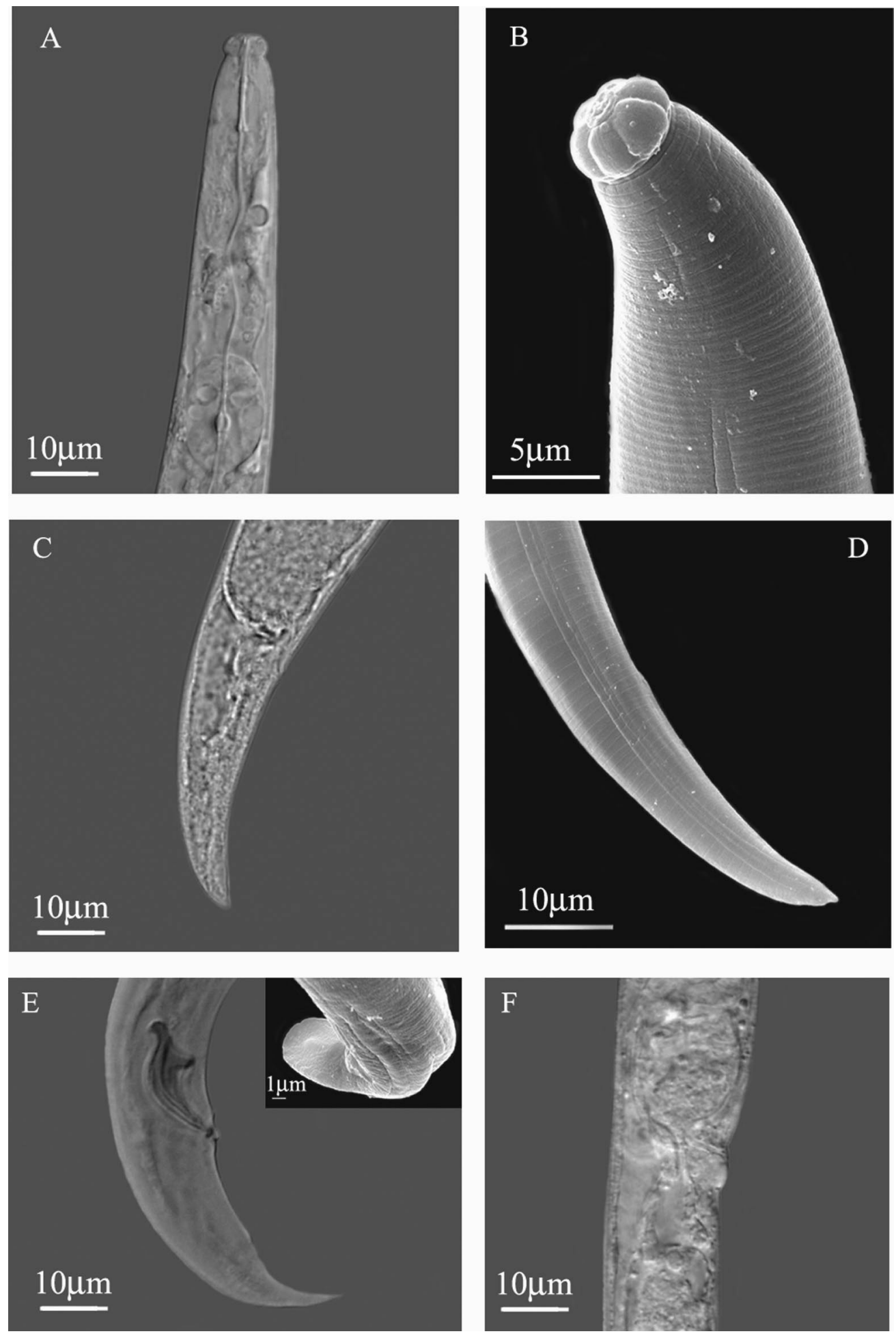

Fig. 6. Bursaphelenchus sexdentati. A: Light micrograph (LM) of anterior region; B: Scanning electron micrograph (SEM) of anterior region; $C$ : LM of female tail; D: SEM of female tail; E: LM of mail tail (stained spicule); inset: SEM of bursa; $F$ : LM of vulval region. 

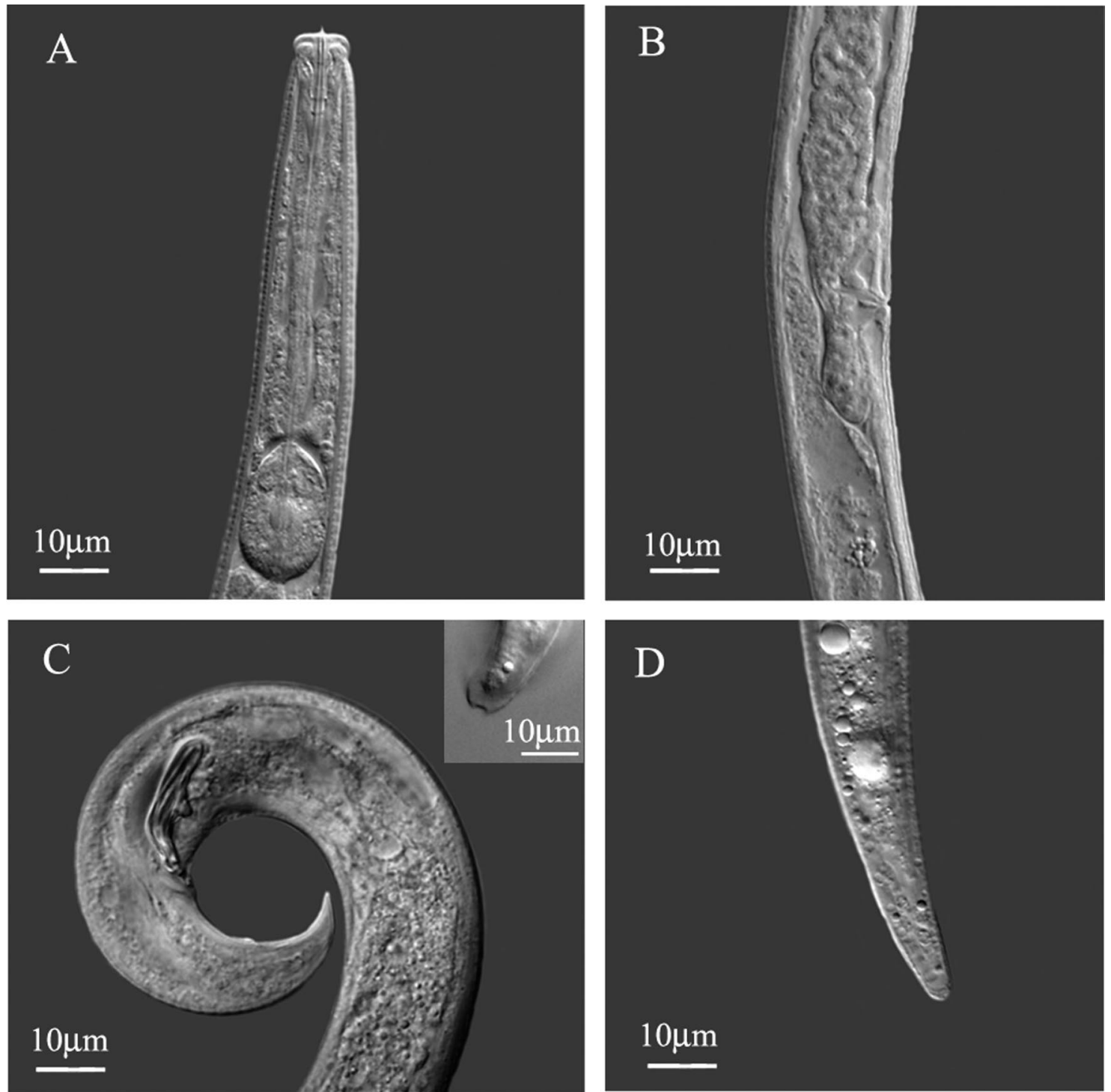

Fig. 7. Bursaphelenchus teratospicularis. A: Light micrograph (LM) of anterior region; B: LM of vulval region; $C$ : LM of male tail; inset: LM of bursa; D: LM of female tail.

culturing to the adult stages and increasing both the speed and reliability of the diagnosis. Furthermore, considering the abundance of rDNA in the genome, this method can be effectively applied to individual nematodes, a significant advantage.

As confirmed by the results of this investigation, ITSRFLP analysis is a more direct approach for identification of Bursaphelenchus species, since specific patterns of restriction fragments have been obtained for each of the five species studied. Although preliminary species determination can be reached by estimation of the number and size of the restriction fragments obtained (Braasch et al., 2001), the use of one reference sample for each species under consideration is strongly recommended. In fact, apart from some inaccuracy that may occur in the estimation of fragment size under different electrophoretic conditions, unoptimised restriction conditions may lead to partial or incomplete digestions making it difficult for correct species allocation of otherwise unidentified isolates. 

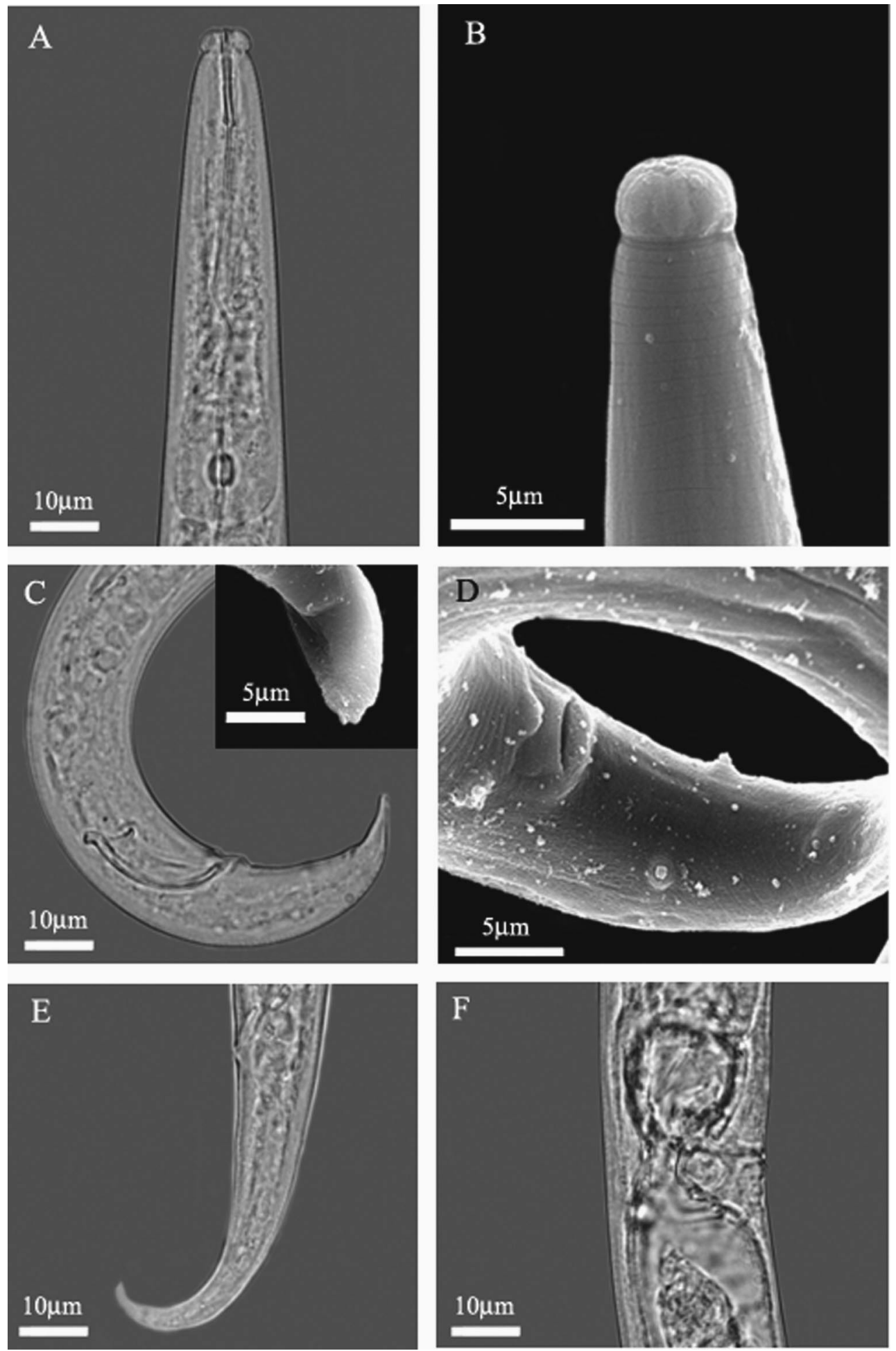

Fig. 8. Bursaphelenchus tusciae. A: Light micrograph (LM) of anterior region; B: Scanning electron micrograph (SEM) of anterior region; C: LM of male tail; inset: SEM of bursa; D: SEM of male tail showing caudal papillae; E: LM of female tail; F: LM of vulval region. 

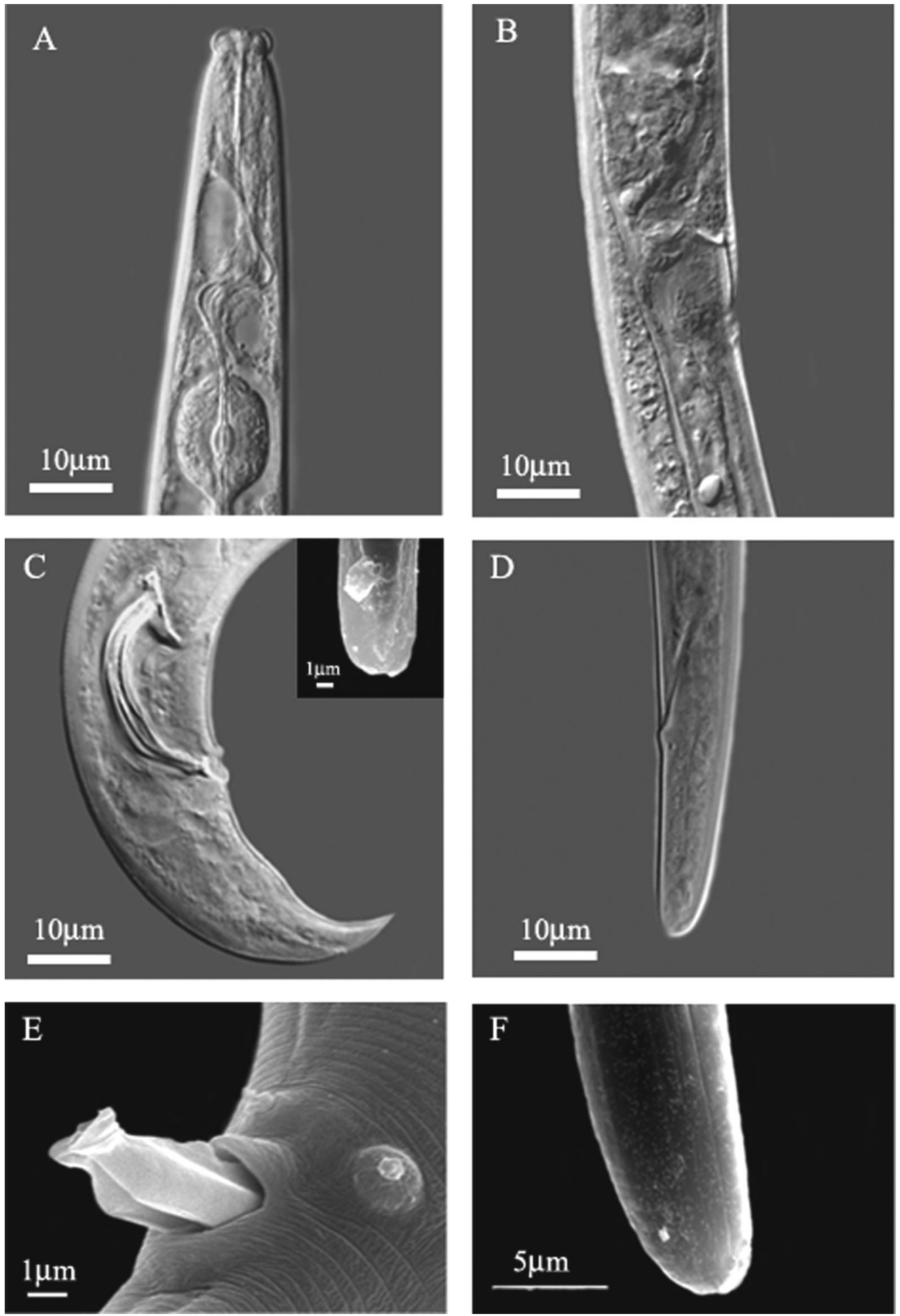

Fig. 9. Bursaphelenchus xylophilus. A: Light micrograph (LM) of anterior region; B: LM of vulval region; C: LM of male tail; inset: Scanning electron micrograph (SEM) of bursa; D: LM of female tail; E: SEM of protracted spicule and cucullus; F: SEM of female tail tip. 

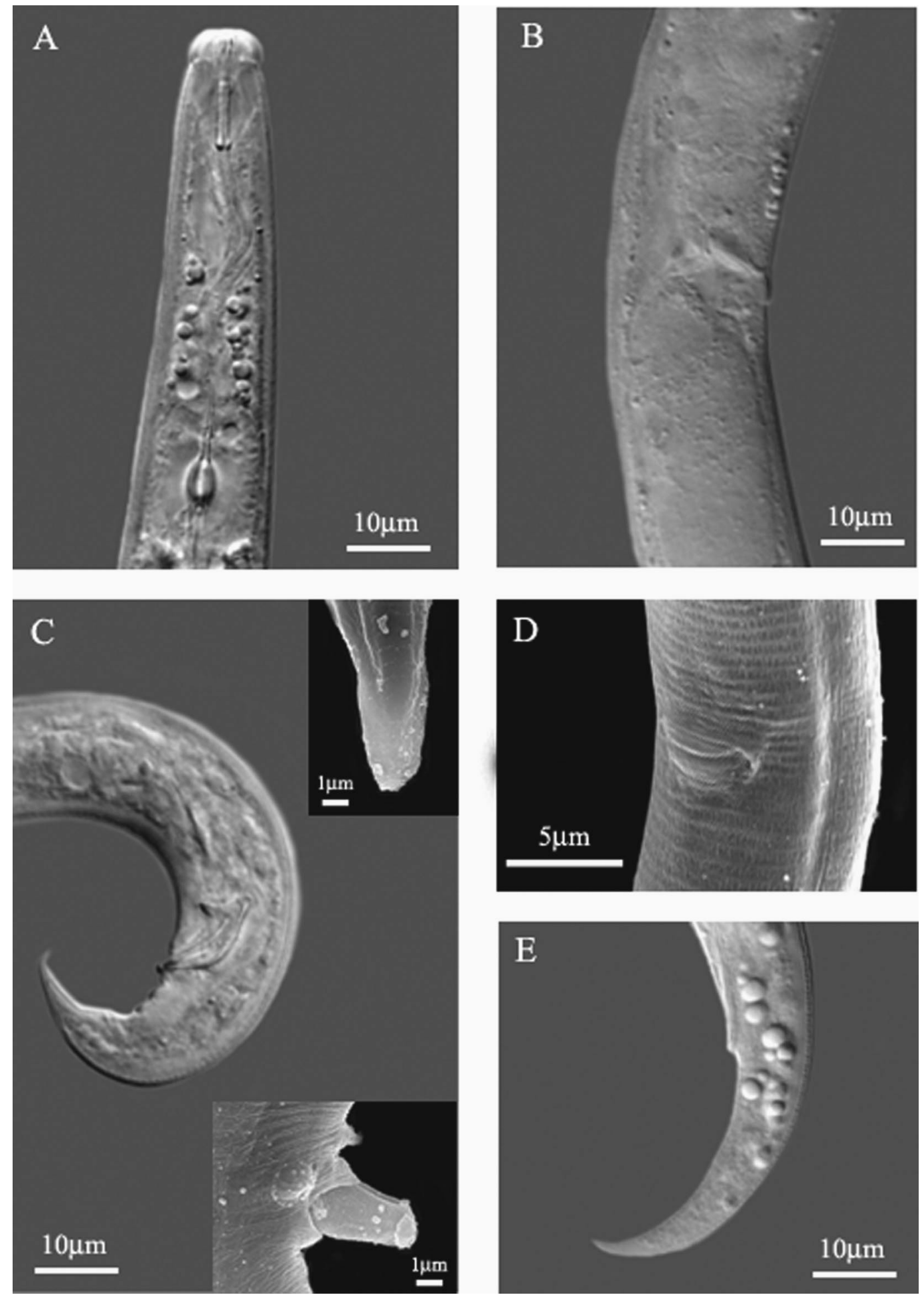

Fig. 10. Bursaphelenchus sp. 1. A: Light micrograph (LM) of anterior region; B: LM of vulval region; C: LM of male tail; inset: Scanning electron micrograph (SEM) of bursa (top) and SEM of spicule protracted and cucullus (bottom); D: SEM of vulval region; E: LM of female tail. 


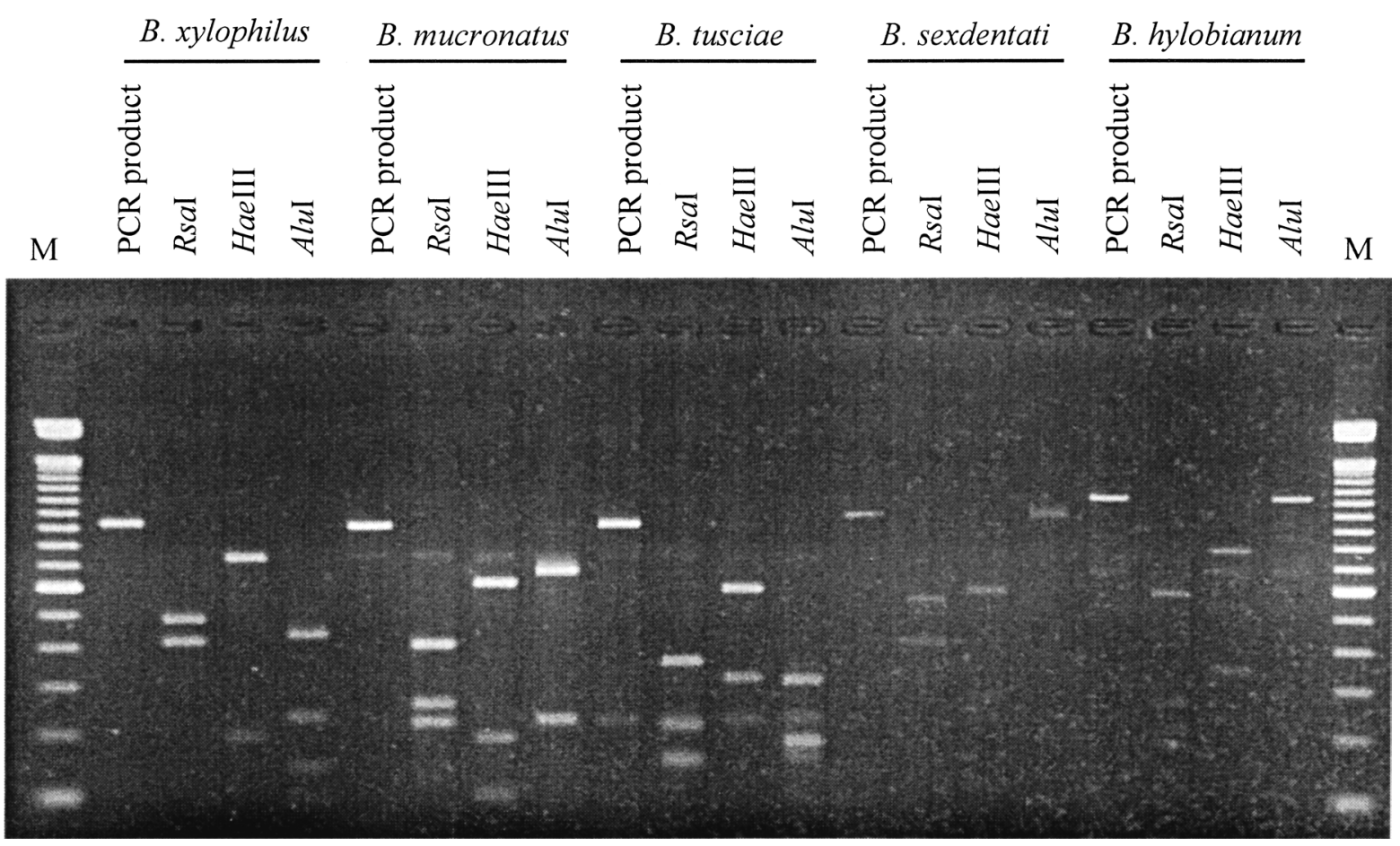

Fig. 11. ITS-RFLP patterns of Bursaphelenchus isolates. Restriction fragments were obtained by digestion of the amplified ITS region of rDNA with the three named enzymes. M: $100 \mathrm{bb}$ Marker (Invitrogene).

Regardless of the underlying structural basis of polymorphism among populations, it is apparent that these genetic differences can discriminate within populations and are convenient diagnostic markers. Overall, ITS-RFLP has been established as a powerful and reproducible tool in the differentiation of many Bursaphelenchus species (Hoyer et al., 1998; Braasch et al., 1999) and has become an important feature in nematode diagnosis. ITS versatility, specificity, ease of experimental manipulation and availability of ITS databases should be increasingly applied in nematology.

This study, based on both morphological and molecular analysis, confirmed the identity of several Bursaphelenchus species reported from Portugal for the first time by Penas et al. (2002). Penas et al. (2002) had identified B. hofmanni from Portugal, although more recent studies have called into question the reliability of this initial identification, the species now being referred to as Bursaphelenchus sp. 1.

The ITS-RFLP patterns, as presented in this paper, were done whenever nematode numbers allowed for such analysis, or when morphological identification was doubtful. A more detailed characterisation of all Portuguese species, including morphology, morphometrics and DNA analysis is being conducted and will soon be published. Bursaphelenchus hylobianum, found for the first time in Europe (Penas et al., 2002) and never previously observed in pine wood from natural stands, was successfully reared in maritime pine branch segments and may therefore be considered as a potential associate of Pinus pinaster. Other Bursaphelenchus species, in addition to B. hylobianum, were found associated with potential insect vectors (unpubl.).

\section{Acknowledgements}

This research was initially supported by grant 11 $189 / 98$ of the Portuguese PRAXIS XXI project, Fundação Ciência e Tecnologia (FCT) and is now currently being supported by a grant from a PARLE D project, (Instituto Nacional de Investigação Agrária (INIA) and FCT) and the European Union 5th Framework, project QLK5-CT-2002-00672 PHRAME (Development of improved Pest Risk Analysis techniques for quarantine pests, using pinewood nematode, Bursaphelenchus xylophilus, in Portugal as a model system). Information regarding 
sampling locations provided by Direcção-Geral das Florestas (DGF) and PROLUNP is hereby acknowledged. This paper is a portion of the $\mathrm{PhD}$ dissertation of the first author, University of Évora. The authors kindly thank Prof. Esperança Pina, Faculdade de Ciências Biomédicas, Universidade Nova de Lisboa, for the use of the scanning electron microscope, as well as Mr Octávio Chaveiro, Estação Agronómica Nacional, for technical assistance.

\section{References}

Ambrogioni, L. \& CARoppo, S. (1998). Morphology and morphometrics of Italian populations of Bursaphelenchus species. Nematologia Mediterranea 26, 97-116.

BRAASCH, H. (2001). Bursaphelenchus species in conifers in Europe: distribution and morphological relationships. Bulletin OEPP/EPPO 31, 127-142.

Braasch, H., Metge, K. \& Burgermeister, W. (1999). [Bursaphelenchus species (Nematoda, Parasitaphelenchidae) found in coniferous trees in Germany and their ITS-RFLP patterns.] Nachrichtenblatt des Deutschen Pflanzenschutzdienste 51, 312-320.

Braasch, H., Tomiczek, C., Metge, K., Hoyer, U., Burgermeister, W., Wulfert, I. \& Schönfeld, U. (2001). Records of Bursaphelenchus spp. (Nematoda, Parasitaphelenchidae) in conifers timber imported from the Asian part of Russia. Forest Pathology 31, 129-140.

BRZESKI, M. \& BAUJARD, P. (1997). Morphology and morphometrics of Bursaphelenchus (Nematoda: Aphelenchoididae) species from pine wood in Poland. Annales Zoologici 47, 305-319.

EISENBACK, J. (1985). Techniques for preparing nematodes for scanning electron microscopy. In: Barker, K.R., Carter, C.C. $\&$ Sasser, N.J. (Eds). An advanced treatise on Meloidogyne, Vol. II. Raleigh, NC, USA, North Carolina State University Graphics, pp. 79-105.
FERRIS, R.V., FERRIS, M.J. \& FAGHIHI, J. (1993). Variation in spacer ribosomal DNA in some cyst-forming species of plant parasitic nematodes. Fundamental and Applied Nematology 16, 177-184.

Hoyer, U., Burgermeister, W. \& BraAsch, H. (1998). Identification of Bursaphelenchus species (Nematoda, Aphelenchoididae) on the basis of amplified ribosomal DNA (ITSRFLP). Nachrichtenblatt des Deutschen Pflanzenschutzdienste 50, 273-277.

Korenchenko, A.E. (1980). [New species of nematodes from the family Aphelenchoididae, parasites of stem pests of the Dahurian Larch.] Zoologichesky Zhurnal 59, 1768-1780.

Mota, M. \& Vieira, P. (EDS). (2004). The pinewood nematode, Bursaphelenchus xylophilus. Proceedings of an International Workshop, University of Évora, Portugal, August 2022, 2001, Nematology Monographs \& Perspectives, Volume 1. Leiden \& Boston, Brill Academic Publishers, 292 pp.

Mota, M., Braasch, H., Bravo, M.A., Penas, A.C., Burgermeister, W., Metge, K. \& Sousa, E. (1999). First report of Bursaphelenchus xylophilus in Portugal and in Europe. Nematology 1, 727-734.

Penas, A.C., Bravo, M.A., Pires, J. \& Mota, M. (2002). Bursaphelenchus species in maritime pine in Portugal. 4th International Congress of Nematology, Tenerife, June 8-13, 2002. Nematology 4, 273. [Abstr.]

RÜHM, W. (1960). Ein Beitrag zur Nomenklatur und Systematik einiger mit Scolytiden vergesellschafteter Nematodenarten. Zoologischer Anzeiger 164, 201-213.

Tarès, S., Abad, P., Bruguier, N. \& de Guiran, G. (1992). Identification and evidence for relationships among geographical isolates of Bursaphelenchus spp. (pinewood nematode) using homologous DNA probes. Heredity 68, 157164.

VRAIN, T. (1993). Restriction fragment length polymorphism separates species of Xiphinema americanum group. Journal of Nematology 25, 361-364. 